\title{
Efficiency of an Electrical Machine in Electric Vehicle Application
}

\author{
S. B. Shah, B. Silwal, A. Lehikoinen \\ Department of Electrical Engineering and Automation, Aalto University, Finland \\ corresponding author: sahas.bikram.shah@aalto.fi
}

\begin{abstract}
Machines have always made life simpler, directly or indirectly. They have been developed for a very wide range of applications. For the performance analysis of any machine, one important parameter to be considered is the machine loss. This consideration has significances like determining the efficiency of the machine which in turn influences the operating cost, determining the heating of machine and for accounting the voltage drops or current component associated with the cause of the losses and many more. Losses in electrical machines can be categorized according to the causes or phenomena that produce them. The efficiency of an electrical machine directly depends on different kind of losses in the machine. Therefore, in this paper we primarily focus on the losses in the machine. First, all possible losses, their causes and effects in an electrical machine have been explained. A brief account of calculating those losses has also been explained. The standard method of calculating the efficiency follows after that. Finally, a finite element analysis is performed for a test machine and the losses and the efficiency of the test machine is studied.
\end{abstract}

Keywords: Electrical Machine, Efficiency Losses, Iron Loss, Mechanical Loss, Resistive Loss, Stray Loss

\section{Introduction}

Traditionally, the losses in electrical machines are divided into the following components

- Resistive losses

- Iron losses

- Mechanical losses

- Stray losses

While this division is somewhat physically inaccurate, it is widely used in related literature and standards, and is therefore also adopted here [19, 21, 12,13]. Shortly, each of the components will be expanded and commented on.

\subsection{Resistive Losses}

Fundamentally, most of the losses occurring in an electrical machine are actually resistive losses. In practice, however, the term is used to denote the ohmic losses in the conductors of the machine $[19,12]$. In synchronous machines, the stator winding is the most significant contributor, while in induction machines the rotor cage will typically also experience large losses. The conventional 
way to calculate the losses is to use the familiar equation,

$$
P_{C u}=R I^{2}
$$

where $I$ is the current flowing in the conductor, and $R$ is its resistance. This equation is indeed accurate if the current density in the conductor is constant. However, in electrical machines this is usually not the case, due to the skin-effect and proximity effects [21]. The former effect will increase the apparent resistance of the conductor, while the latter may result in "invisible" resistive losses. These factors will be dealt with more closely later on. Furthermore, the resistivity of typical conductor materials is temperature dependent. Therefore, the operating point of the machine has to be taken into consideration as well. Due to these complications, a simplified approach is usually taken. The resistance is either measured using a DC current supply [12], or it is calculated based on conductor geometry and material. Resistive losses are then calculated from the DC resistance and current values, and all extra losses due to the aforementioned effects are lumped under stray losses. Calculated this way, the resistive losses typically make up roughly $50 \%$ of the total losses, depending on the machine size and type [14].

\subsection{Iron Losses}

Iron losses are power losses taking place in the magnetically active iron parts of the machine due to the time-varying flux density. It must be noted that their exact nature and characteristics are being intensively studied even at the moment, and it seems that a lot is still to be discovered and verified. Nevertheless, one of the most widely accepted models is briefly presented here on a conceptual level [2].

The iron losses can be divided into eddy current, hysteresis and excess losses. Of these three, the eddy current losses are probably the easiest one to understand. Simply put, a changing flux density will induce a rotational electric field, which will in turn create currents in the iron, causing resistive losses inside the iron. The magnitude of these losses is strongly dependent on the size of the continuous conductive regions. For this reason, electrical machines are usually assembled from thin iron sheets, insulated from each other. In this case, the eddy current losses will be roughly proportional to the square of the sheet thickness, flux density and frequency, and linearly proportional to their conductivity. The hysteresis and excess losses are more related to the microscopic material properties of iron. The iron sheets are assumed to consist of small magnetic domains, which change their size according to the external magnetic field. The changes in domain sizes result in two different kinds of microscopic eddy currents near the domain walls, causing the hysteresis and excess losses. Of these two, the hysteresis losses are roughly proportional to the frequency and the square of the flux density, while the excess losses are often assumed to be proportional to the $1.5^{\text {th }}$ power of both.

The hysteresis losses are especially difficult to calculate, since they depend not only on the instantaneous flux density, but also on its history. In practice however, these effects are often ignored and the iron losses are calculated with the simplified formula

$$
P_{F e}=\left(c_{\text {hyst }} f+c_{\text {eddy }} f^{2}\right) B^{2}+c_{\text {excess }}(f B)^{1.5}
$$

where $c_{\text {hyst }}, c_{\text {eddy }}$ and $c_{\text {excess }}$ are the loss coefficients for the corresponding loss components. The formula gives the iron losses per unit volume or unit mass, depending on how the coefficients are defined. The loss coefficients are typically obtained from the manufacturer of the iron sheets, or by 
self-performed measurements. Depending on the type of iron used, the exponents of frequency and flux density related to hysteresis and excess losses may also change slightly.

Another complicating facture related to iron losses is the manufacture process of the electrical machine. While the electrical steel sheets are cut or punched into their final geometry, the microstructure of the steel is deformed near the cutting edges. Furthermore, the manufacture process in general creates additional mechanical stresses inside the iron. Both these factors will influence the magnetic properties of the iron, including its loss characteristics. While these factors typically increase the iron losses, precise changes and their mechanisms are yet to be determined.

\subsection{Mechanical Losses}

The mechanical losses encompass, as their name suggests, the losses due to friction in bearings, air drag on the rotating parts and cooling fans, and other losses inherently mechanical in nature. Of these, the aerodynamic losses depend strongly on the internal structure and geometry of the machine, and are perhaps the most difficult to calculate [21, 12], [14]. Their magnitude and significance depend on the nature of the machine in question: in high-speed machines, the outer surface of the rotor can move at almost the speed of sound, and will experience significant drag.

Fortunately, the mechanical losses are almost independent of the loading of the machine. Thus, they are often determined from the results of the no-load test, from the intersection of the extrapolated no-load power curve with the $\mathrm{U}=0$ line voltage [12]. The feasibility of this approach has been repeatedly questioned, however.

\subsection{Stray Losses}

The stray losses are a contradictory loss component, often making up as much as one fifth of the total losses [16]. By definition, stray losses make up the difference between measured total losses and approximated resistive, mechanical and iron losses $[12,13]$. In other words, they appear to only consist of losses of anomalous origins. However, they do actually have some well-known and well-understood components [16, 22]. First of all, all skin effect losses are defined to fall under stray losses. In a solid conductor carrying a time-varying current, the current density will not be constant across the conductor cross-section. Instead, the current will usually tend to focus near the conductor surface (conductor in air) and the air-gap (actual conductor in a machine). This will increase the apparent resistance of the conductor, increasing the resistive losses. Several wellknown formulae to calculate the skin-effect losses for different types of conductors and windings can be found in the literature $[8,10]$. Approximate methods also exits, based on dividing the conductor into thin parallel-connected sheets $[19,21]$.

To prevent high skin-effect losses, large solid conductors are very often split into several insulated sub-conductors, connected in parallel. This is very effective in reducing the skin-effect losses, but can often create so-called proximity effect or circulating current losses. These losses come about because the exact positioning of the small sub-conductors in the slots cannot be controlled exactly. This approach typically results in different voltages being induced on different conductors. Since the sub-conductors are connected in parallel, this results in currents circulating between them, making up for the difference in voltages. These circulating currents may be very difficult to detect from the measured total currents. Furthermore, they may increase the resistive losses of the windings by up to $50 \%$ in some high-speed machines [15]. Finally, estimating and predicting 
them is very difficult due to the randomness inherent in the problem. Indeed, they are a subject of intensive study in the research group of electro mechanics in Aalto University.

Furthermore, some iron losses are also included in the stray losses. The so-called iron losses of the machine are usually not determined directly. Instead, they calculated from no-load test results, and assumed to stay the same in full-load operation as well [12]. While, it is indeed true that the fundamental flux density will be almost the same in a loaded and non-loaded machine, the actual flux density distribution (w.r.t. to position and time) near the air-gap region will not [16]. The flux density in the air-gap can be calculated from the product of the air-gap permeance $\Lambda$ and the magnetomotive force $F$.

$$
B(x, t)=\Lambda(x, t) F(x, t)
$$

Of these two, the permeance depends on the machine geometry, while the magnetomotive force depends on both the geometry and instantaneous currents of the machine. The air-gap permeance will not be constant due to the slotting in the stator and rotor, and the rotation of the rotor. Neither will the magnetomotive force, due to the discrete locations of the conductors and the time-varying currents. As can be seen, flux density in the air-gap will be a complicated function of position and time. Specifically, the magnetomotive force distribution at full load will be hugely different from the distribution at no-load. This effect can be further compounded by the complicated interaction between the magnetomotive force and the air-gap permeance. Therefore, even though the fundamental flux density will remain relatively the same, its higher harmonics (with respect to both position and time) will not $[16,11]$. These flux density harmonics will penetrate into the stator and rotor iron, causing eddy-current, hysteresis and excess losses. The magnitude of these losses can be calculated with the approximate loss equation defined earlier, by substituting the corresponding amplitudes and frequencies of the flux density harmonics $[16,6]$.

However, the air-gap flux density harmonics can also cause resistive losses in several different ways. In induction machines or synchronous machines with a damper winding, the harmonics will induce voltages in the rotor winding. These voltages, in turn, can create currents flowing in the winding [1]. Since the frequency of the harmonics will usually be high, the resistive losses due to these currents will be compounded by the strong skin-effect phenomenon. However, these currents will in turn have their own associated magnetomotive force distribution, damping some flux density harmonics and amplifying others. This extremely complicated interaction has to be taken into account when trying to obtain accurate loss values from analytical calculations. In large induction machines, more than $50 \%$ of the total stray losses can be caused by these rotor currents $[16,1]$. Furthermore, the air-gap harmonics can also create circulating currents in the stator winding, provided that it has several parallel sub-conductors. Finally, they can also cause eddy-current losses in any conductor too near the air-gap, since in this case the harmonic flux will pass through the conductor and induce rotation electric fields into it.

\subsection{Other Losses}

There are also several other loss components, which do not conveniently fall into any of the aforementioned categories and which are often ignored. For instance, iron losses due to the end-winding flux, while stray losses in the literal sense, are very often completely ignored due to the three-dimensional nature and the associated computational complexity of the problem. Likewise, losses due to the leakage flux passing through the machine frame are most often ignored. Admittedly, these losses are typically quite small, so their exclusion does not present a large error 
[18]. Three-dimensional finite element methods are considered the only way to calculate these losses accurately."

Currents flowing between the iron sheets in the machine core are another complicated source of losses. These losses are related to the manufacture of the machine. The iron sheets are usually punched into their final shape: a process which creates burrs in the cut edges. These burrs create electrical contacts between the sheets, resulting in currents circulating in the machine core, and therefore resistive losses. These losses can, at worst, be very significant, and are known to have caused generator meltdowns in extreme cases [17]. Also these losses are extremely random in nature, and are currently being studied.

Finally, exclusive to permanent magnet machines are the losses due to eddy currents in permanent magnets. Permanent magnets often present a large volume of relatively high conductivity. Therefore, any change in the flux density in the magnets will result in relatively large eddy current densities and associated losses. Furthermore, these losses can increase the temperature of the magnets up to a point where the magnet characteristics start to deteriorate [3]. Although analytical formulae for these eddy currents and losses do exist, finite element methods are often used for increased accuracy.

\section{Efficiency of an Electric Machine}

One of the components that provides drive EHV is an electric motor. The requirement of the electric vehicle is fast start/stop operation. Electrical vehicle should have certain torque speed characteristic. Then, overall desire in designing electrical machine should be to obtain high efficiency in that operation range.

The general torque speed characteristic of an electrical machine can be seen in Figure 2. Due to different structure and operating principle of electrical machines different machines are used for different operating range. The efficiency map of different electrical machine is shown in Figure 1 and Figure 2. An electric motor operates in constant torque region till its base speed and if one wants to get higher speed than the base speed, field weakening have to be done and then motor operates in constant power region. There is tradeoff between torque and speed and depending on the required speed range, an efficient machine can be designed.

There are many factors that influence the efficiency of an electrical machine. Losses in electrical machine are one of them. The different losses in electrical machines are already discussed. The general graph showing the efficiency and different loss as different load is shown in Figure 2. However, the losses are not only important factor when determining the efficiency. The other factor such as power supply quality and its harmonics, over sizing of the system, transmission and mechanical components plays significant role in determining the efficiency of machine [7]. 


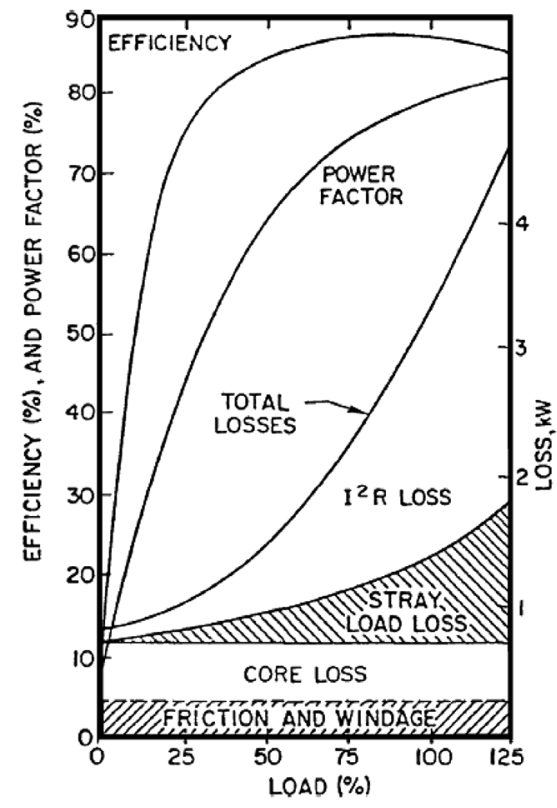

Fig. 1. Losses and Efficiency as a function of load [18]

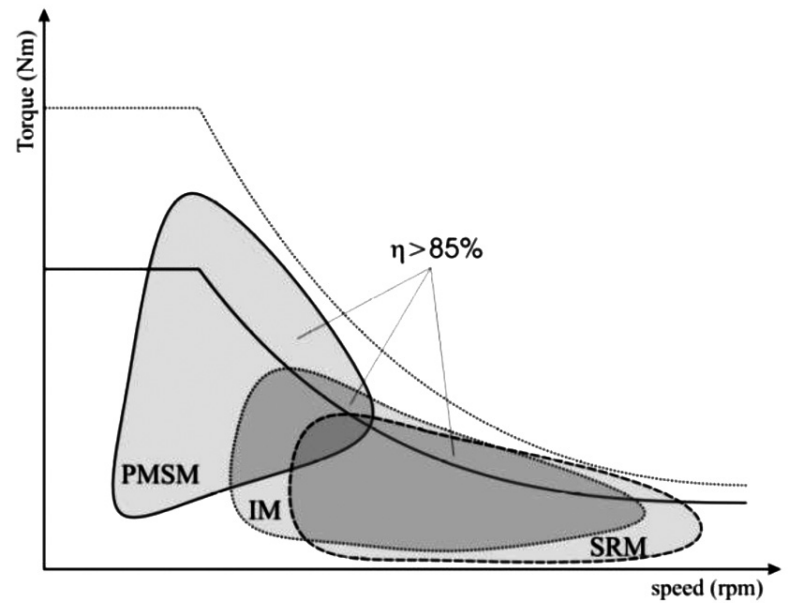

Fig. 2. Efficiency map of different electrical machines [19]

The electric motors are designed for optimal power supply and deviations from such power supply will deteriorate the efficiency of the machine. The slight deviation of the phase supply of $2 \%$ can increase the loss up to 25\% [9] and reduce the efficiency of the machine. Moreover, electric motors in electric vehicles are supplied with converters and the ac supply obtained from those converters is not purely sinusoidal and contains multiples of fundamental frequency called harmonics. It increases the losses and noise, reduces the torque and causes overheating and hence reduces the life of the machine. The other important aspect is the cost of the machine. It can be seen from the figure that machine efficiency peaks at around $75 \%$ of load. The unnecessarily over sizing and excessive use of material should be avoided. It should be optimized in such a way that it meets torque speed range and remains within the desired cost 


\section{Methods of efficiency calculation}

There are certain IEC standards that define how to measure efficiency of an electrical machine. Two methods are discussed briefly.

\subsection{Direct Method (IEEE 112-B, CSA-390)}

Mechanical power can be calculated by directly measuring the torque and speed of the machine. Similarly, electrical power can be obtained by measuring voltage, current and their phase angles. The analytical approach is to calculate its torque and other parameters accurately using mathematical formulation and finite element method. The general formula to calculate efficiency from direct method is given below.

$$
\text { Efficiency }(\eta)=\frac{\text { Mechanical Output power }}{\text { Electrical input power }} \times 100 \%
$$

\subsection{Indirect Method (IEC 34-2)}

The indirect method avoids the requirement of experimental setup and transducers to measure torque and speed. It can be obtained by determining electrical input power and losses. There are various methods to determine losses in electrical machines analytically and experimentally [5] [20]. Extensive research is going on to compute the losses accurately in electrical machines [23] [4].

$$
\text { Efficiency }(\eta)=\frac{\text { Electrical input power }- \text { Losses }}{\text { Electrical input power }} \times 100 \%
$$

\section{Finite Element Analysis}

With recent developments in the field of computing resources, the Finite Element Analysis (FEA) is gaining immense popularity, especially in the design and analysis of an electrical machine. The main idea of Finite Element Analysis is to divide a large and complex problem area into a mesh of small and simple problem areas. The small problem area is defined as an element. Then a solution is approximated in each small element using a function, generally a polynomial. The fundamentals of electromagnetic fields and space and time relationship in the case of electrical machine can be well described by Maxwell's equations. The magnetic vector potential formulation is the most commonly used formulation in the numerical analysis of electrical machine. The magnetic vector potential is defined as the curl of the magnetic flux density $\boldsymbol{B}$,

$$
\boldsymbol{B}=\nabla \times \boldsymbol{A}
$$

Substituting (4) to one of the Maxwell's equation, and again substituting the resulting equation into one of the other Maxwell's equation , we obtain the partial differential equation

$$
\nabla \times v \nabla \times \boldsymbol{A}=\boldsymbol{J}
$$

The change in magnetic field induces an electric field which in turn produces the eddy current. From Maxwell's equation and (4) we obtain, so that the electric field strength $\boldsymbol{E}$ is given by, 


$$
\boldsymbol{E}=-\frac{\partial \boldsymbol{A}}{\partial t}-\nabla \phi
$$

Since, the current density, from (5) and (6) we have

$$
\nabla \times v \nabla \times \boldsymbol{A}=-\sigma \frac{\partial \boldsymbol{A}}{\partial t}-\sigma \nabla \phi
$$

The current density corresponding to (7) is the eddy current density as it is produced by the electric field which is induced by the change in the magnetic field, so (7) is valid for eddy current regions. But, the current density corresponding to (5) is the source current density that is the current density in the source coils and windings. So, both equations (5) and (7) are combined to give a single equation which corresponds to the whole problem region which is expressed as given in (8).

$$
\nabla \times v \nabla \times \boldsymbol{A}+\sigma \frac{\partial \boldsymbol{A}}{\partial t}+\sigma \nabla \phi=\boldsymbol{J}_{s}
$$

where, $v$ is the reluctivity of the material, $\sigma$ is the conductivity of the medium and $\boldsymbol{J}_{s}$ is the current density of the source.

An induction machine with the parameters given in Table I is used as a test machine in this paper. We perform a Finite Element Analysis for the given test machine and study how different losses behave as the load of the machine is increased. The resistive and the core losses in both the stator and the rotor are particularly in our interest. The losses and the efficiency are plotted with respect to the slip of the machine. Higher loads mean higher slip and lower speed of the machine. Figure 3 shows the resistive and the core losses in the stator and the rotor. The resistive losses increase significantly as the loading increases because with more loads, the machine draws more current which contributes to the load. The core losses remain more or less steady. Figure 4 shows the plot of efficiency when the load is increased. The efficiency reduces to below $10 \%$ when the slip is 50 times the rated slip. Indirect method is used to calculate the efficiency.

Table 1. Test Machine Parameters

\begin{tabular}{|c|c|}
\hline Parameter & Value \\
Number of poles & 4 \\
Connection & Star \\
\hline Rated Voltage [V] & 400 \\
\hline Supply frequency [Hz] & 50 \\
\hline Rated Current [A] & 70 \\
\hline Rated Torque [Nm] & 260 \\
\hline Rated Power [kW] & 37 \\
\hline
\end{tabular}




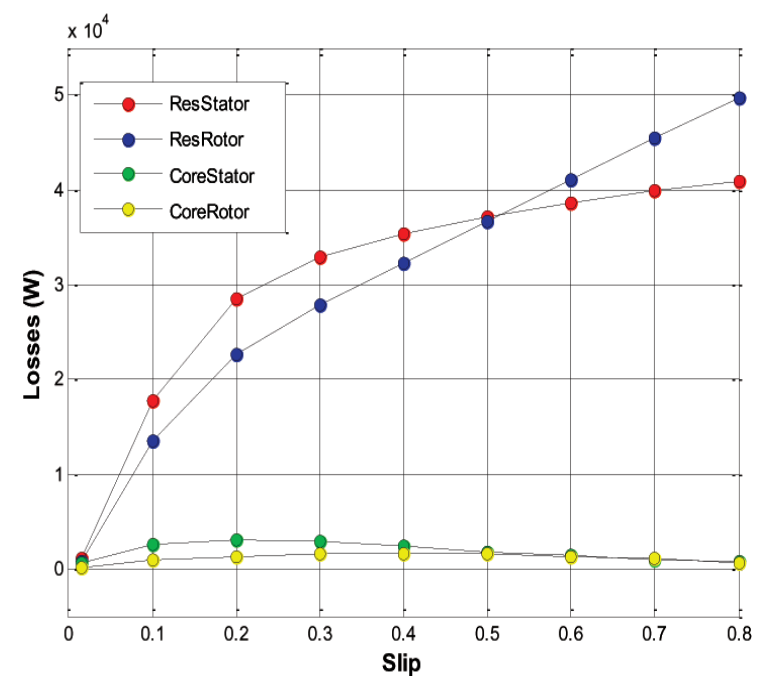

Fig. 3. Losses with respect to the slip of the machine

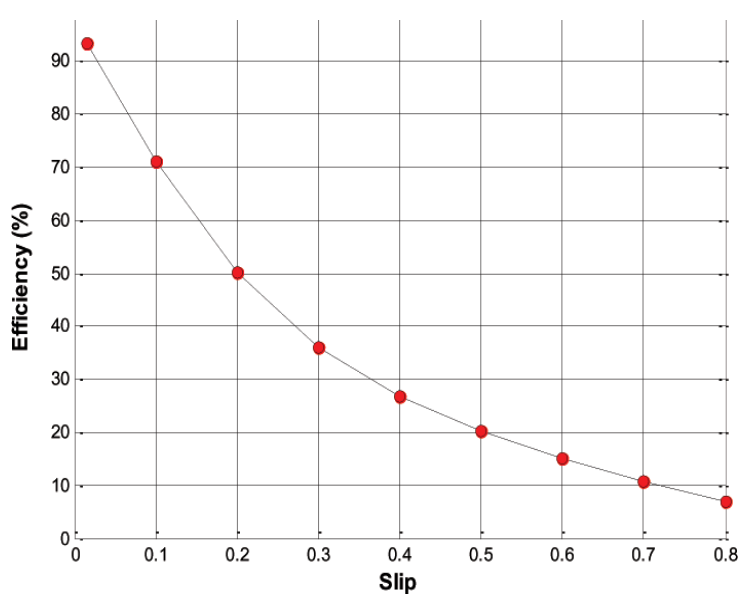

Fig. 4. Efficiency of the machine with respect to the slip

\section{Conclusion}

In electric vehicle application, designer has an advantage to choose from different types of electric machines. Based on literature study, choice of electric machine depends on the topology of the machine. The DC current machine have low initial cost but high maintenance cost and it offers low power density and bad efficiency for hybrid electric vehicle. Induction machines are cost efficient and have good efficiency over a high speed range. Switch reluctance machine is comparable to induction machine in terms of power density and cost. The disadvantage is it has torque ripples. PMSM has an advantage of high power density and has good efficiency in defined speed range. Hence, electric machine can be exploited to its full potential if used carefully. For example, in hybrid vehicle, if it is series hybrid system then the machine should be designed for maximum power and full speed range then induction machine is suitable but in parallel hybrid which are usually limited to low speed PMSM are suitable. 


\section{References}

[1] Alger PL (1957), Induced high-frequency currents in squirrel-cage windings. Transactions of the American institute of electrical engineers, power apparatus and systems 76:724-729.

[2] Ansys Inc (2012), Simulation of permanent magnet machines. Seminar material, Helsinki.

[3] Arkkio A (2012), Lecture material for the course Special course on electromechanics. Aalto University School of Electrical Engineering, Espoo.

[4] Bertotti G (1985), Physical interpretation of eddy current losses in ferromagnetic materials. Journal of applied physics 57: 2110-2117.

[5] Burais N (1981), Iron losses calculation in non-oriented steel plate. IEEE transactions on magnetics 17: 2577-2579.

[6] Christofides N (1965), Origins of load losses in induction motors with cast aluminium rotors. Proceedings of the institution of electrical engineers 112: 2317-2332.

[7] De Almeida AT and Ferreira F (1997), Efficiency testing of electric induction motors. Springer Verlag, Germany, 1997.

[8] Field AB (1905), Eddy currents in large slot-wound conductors. Transactions of the American Institute of Electrical Engineers 14:761-788.

[9] Finken T and Hameyer K (2008), Comparsion and design of different electrical machine types regarding their applicability in hybrid electric vehicles. Proceedings of the 2008 International conference on electrical machines 1-5.

[10] Gilman RE (1920), Eddy current losses in armature conductors. Transactions of the American Institute of Electrical Engineers 39: 997-1056.

[11] Heller B and Hamata V (1977), Harmonic field effects in induction machines. Elsevier Scientific Publishing Company, Amsterdam.

[12] IEC 60034-2-1. "Rotating electrical machines Part 2-1: Standard methods for determining losses and efficiency from tests (excluding machines for traction vehicles)," Geneva, Switzerland, IEC, 2007.

[13] IEEE Std 112-2004 "IEEE standard test procedure for polyphase induction motors and generators." New York, The Institute of Electrical and Electronics Engineers, Inc., 2004.

[14] Jokinen $\mathrm{T}$ (2014), Reducing losses of electrical machine, $14^{\text {th }}$ international symposium. Topical problems in the field of electrical and power engineering.

[15] Lähteenmäki J (2002), Design and voltage supply of high-speed induction machines. Acta Polytechnica Scandinavica, Electrical Engineering series, 108, Espoo.

[16] Lehikoinen A (2013), Calculation and measurement of stray load losses in induction machines. Master's thesis, Espoo.

[17] Lehikoinen A (2011), Sähkölevyjen välisten oikosulkujen havainnointimenetelmät sähkökoneissa. Bachelor's Thesis, Espoo, Finland.

[18] Lin R, Haavisto A and Arkkio A (2010), Analysis of eddy-current loss in end shield and frame of a large induction machine. IEEE transactions on magnetics 46: 942-948.

[19] Lipo TA (2007), Introduction to AC machine design. Madison WI, University of Wisconsin.

[20] Luomi J (1993), Finite element method in electrical machines. Gothenburg, Sweden.

[21] Pyrhönen J, Jokinen T and Hrabovcová V (2008), Design of rotating electrical machines. Chichester, Wiley.

[22] Schwarz KK (1964), Survey of basic stray losses in squirrel-cage induction motors. Proceedings of the institution of electrical engineers 111: 1565-1574.

[23] Shah SB (2013), Finite element modelling of additional losses due to inter-laminar short-circuit current in electrical machines. Aalto Univeristy, Espoo, Finland. 